\title{
A Study on Incidence, Etiology and Outcome of Different Type of Cerebral Stroke in Tribal and Non Tribal Population of Jharkhand
}

\author{
Dr. Vidyapati ${ }^{1}$,Dr. SanjeevKumar Khunte ${ }^{2}$,Dr. DiwakarKumar ${ }^{3}$, \\ Dr. Jitendra Kumar ${ }^{4}$ \\ ${ }^{1}$ Associate Professor, Department Of Medicine, RIMS, Ranchi \\ ${ }^{2}$ Junior Resident , Department Of Medicine, RIMS, Ranchi \\ ${ }^{3}$ Senior Resident, Department Of Medicine, RIMS, Ranchi \\ ${ }^{4}$ Junior Resident, Department Of Medicine, RIMS, Ranchi
}

\begin{abstract}
Cerebrovascular accident is a global health problem and leading cause of mortality and morbidity worldwide. Prevalence rates reported for cerebrovascular accident (CVA) worldwide vary between 500 to 800 per 100,000 population. They account to about 50\% of all neurological hospital admission.Due to increase in burden of stroke in coming years and limited availability of stroke care in India, it would be better to study on preventive measures that will reduce incidence of stroke.The objective of our study was to assess the clinical profile,risk factors and the prognosis of cerebrovascular accident in 100 patients(50 Tribal and 50 Non Tribal patients) presented with cerebrovascular accident.These patient were subjected to detailed history, physical examination,ct/mri of brain and placed into one of two category of stroke either ischemic(thrombotic or embolic) or haemorrhagic.In the present study cerebrovascular strokes was more common in males(65\%) than females(35\%).Most common age group was 50-to 59 year (35\%) followed by60-69 year(27\%)and most common etiology was cerebral hemorrhage (56\%).Most common risk factor was hypertension 61\%,alcoholism $33 \%$,smoking $23 \%$, hyperlipidemia $20 \%$ and diabetes $21 \%$.The overall clinical diagnosis correlation with ct scan brain was $90 \%$ accurate.In hemorrhagic stroke common site was basal ganglia(especially the putamen) followed by thalmus followed by internal capsule and periventriculer area.Most commoninvolved area was mca(middle cerebral artery) territory $80 \%$, overall mortality was $35 \%$ which was higher in hemorrhagic stroke $52 \%$ as compared to ischemic stroke $18 \%$.
\end{abstract}

Keywords: Cerebral stroke, Tribal and non Tribal population, incidence, etiology and outcome

\section{Introduction}

Cerebrovascular accident is a global health problem and leading cause of mortality and morbidity worldwide. Prevalence rates reported for cerebrovascular accident (CVA) worldwide vary between 500 to 800 per 1,00,000 population. They account to about 50\% of all neurological hospital admission. The 1990 Global Burden of Disease (GBD) study provided the first global estimate on the burden of 135 diseases, and cerebrovascular diseases ranked as the second leading cause of death after ischemic heart disease. In 2001 it was estimated that cerebrovascular diseases (stroke) accounted for 5.5 million deaths worldwide, equivalent to $9.6 \%$ of all deaths. Two-thirds of these deaths occurred in people living in developing countries and $40 \%$ of the subjects were aged less than 70 years. Additionally, cerebrovascular disease is the leading cause of disability in adults and each year millions of stroke survivors has to adapt to a life with restrictions in activities of daily living as a consequence of cerebrovascular disease. Risk factors for CVA are divided into modifiable and non modifiable risk factors. Non modifiable risk factors include age, sex, ethnicity and geography, family history of stroke. Modifiable risk factors are smoking, alcohol consumption, drug abuse, arterial hypertension,diabetes mellitus,dyslipidemia, and heart disease.Effective risk factor intervention offers a real hope of reducing stroke mortality and morbidity.Due to increase in burden of stroke in coming years and limited availability of stroke care in India, it would be better to study on preventive measures that will reduce incidence of stroke. An attempt has been made in this study to know the prognosis of CVA with reference to risk factors in rural population.

The World Health Organization (WHO) definition of stroke is: "rapidly developing clinical signs of focal (or global) disturbance of cerebral function, with symptoms lasting 24 hours or longer or leading to death, with no 
apparent cause other than of vascular origin". By applying this definition transient ischemic attack (TIA), which is defined to last less than 24 hours, and patients with stroke symptoms caused by subdural hemorrhage, tumors, poisoning,ortrauma are excluded.The pathological background for stroke may either be ischemic or hemorrhagic disturbances of the cerebral blood circulation

Ischemic stroke (infarction): Thrombotic cerebral infarction results from the atherosclerotic obstruction of large cervical and cerebral arteries, with ischemia in all or part of the territory of the occluded artery. This can be due to occlusion at the site of the main atherosclerotic lesion or to embolism from this site to more distal cerebral arteries. Embolic cerebral infarction is due to embolism of a clot in the cerebral arteries coming from other parts of the arterial system, for example, from cardiac lesions, either at the site of the valves or of the heart cardiac cavities, or due to rhythm disturbances with stasis of the blood, which allows clotting within the heart as seen in atrial fibrillation.

Hemorrhagic stroke Spontaneous intracerebral hemorrhagesare mainly due to arteriolar hypertensive disease, and more rarely due to coagulation disorders, vascular malformation within the brain, and diet (such as high alcohol consumption, high blood cholesterol concentration, high blood pressure, etc.). Cortical amyloid angiopathy (a consequence of hypertension) is a cause of cortical hemorrhages especially occurring in elderly people and it is becoming increasingly frequent as populations become older.

\section{Aims And Objectives}

The objective of our study was to assess the clinical profile, risk factors and the prognosis of cerebrovascular accident in 100 patients(50 Tribal and 50 Non Tribal patients) presented with cerebrovascular accident. As few data were available on CVA in rural population, this study is conducted to identify the risk factors, etiology and outcome in patients with CVA.

\section{Material And Methods}

This Prospective observational study was carried out in 100 patients(50 Tribal and 50 Non Tribal) with Cerebrovascular accident (CVA), who were admitted in department of General Medicine in RAJENDRA INSTITUTE OF MEDICAL SCIENCE, RANCHI betweenMarch2016to march2017. Ethics committee approval was taken for the study.

The patients were seprated on a clinical basis and investigation and placed into one of the following two categories of cerebrovascular disease:ischemic (thrombotic or embolic stroke) and hemorrhagic stroke.

\section{Inclusion criteria:}

1.All patients having clinical and radiologic confirmed diagnosis of stroke were included.

2.Age 20 yrs or above were included in this study.

Exclusion criteria:

1.Age less than $20 y$ rs

2.Patients having stroke due to trauma.

3. Patients with Cortical venous thrombosis.

4.Patients presented with transient ischemic attack.

On admission, the following investigation were carried out:A complete hemogram ,routine urine,random blood suger,liver function test,kidney function test,lipid profile,ecg, $\mathrm{x}$ ray chest ,echocardiography,brain imaging (CT/MRI),coagulation profileand carotid Doppler.

\section{In this study}

1. Patients with $\mathrm{HbA} 1 \mathrm{C}>6.5 \%$ and patients who were on anti-diabetic medication were considered under risk factor for diabetes mellitus.

2. Patients who were known hypertensive prior to the onset of CVA and newly detected patients with evidence of hypertensive retinopathy were considered under risk factor for hypertension.

3. Dyslipidemia was diagnosed as per American Heart Association (AHA) guidelines (2011) as serum cholesterol > $200 \mathrm{mg} / \mathrm{dl}$, low density lipoprotein $(\mathrm{LDL})>130 \mathrm{mg} / \mathrm{dl}$, triglycerides $>150 \mathrm{mg} / \mathrm{dl}$ and high density lipoprotein $(\mathrm{HDL})<40 \mathrm{mg} / \mathrm{dl}$.

4. Patients with ischemic heart disease, congestive heart failure, rheumatic heart disease, atrial fibrillation, corpulmonale, and cardiomyopathy were considered as suffering from heart disease.

5. Smoking (who were currently smoking more than 10 cigarettes per day for more than 6 months) and alcohol intake (> 3 standard drinks/day for a minimum of 6 months) were based on clinical history of consumption of these substances.

6. Past history of Transient ischemic attack(TIA) and stroke was considered if the patient had any symptoms suggestive of TIA or stroke in the past. 


\section{Observation And Results}

100 cases (50 Tribal and 50 Non Tribal) of cerebrovascular stroke were studied in medical ward of RAJENDRA INSTITUTE OF MEDICAL SCIENCE,RANCHI during a period of 1.3.2016 to 30.3.2017 to evaluate the clinical profile and frequency of risk factors. Risk factors for CVA like hypertension, diabetes, dyslipidemia ,family history of CVA was evaluated.

Table - 1 Risk factors for cerebrovascular disease

\begin{tabular}{|c|c|c|c|c|c|c|c|c|c|}
\hline \multirow[t]{2}{*}{ Risk factor } & \multicolumn{2}{|c|}{ Infarct } & \multicolumn{2}{|c|}{ Haemorrhages } & \multicolumn{2}{|c|}{ Total } & \multirow{2}{*}{ Total } & \multirow{2}{*}{$\begin{array}{l}\text { Present } \\
\text { Study\% }\end{array}$} & \multirow{2}{*}{$\begin{array}{l}\text { L.pinhero } \\
\text { et al \% }\end{array}$} \\
\hline & $\mathrm{M}$ & $\mathrm{F}$ & $\mathrm{M}$ & $\mathrm{F}$ & $\mathrm{M}$ & $\mathrm{F}$ & & & \\
\hline Age $>50 y r s$ & 20 & 16 & 30 & 13 & 50 & 29 & 79 & $79 \%$ & $78 \%$ \\
\hline Sex & 25 & 19 & 40 & 16 & 65 & 35 & 100 & $65 \%$ male & 74 \\
\hline Hypertension & 12 & 9 & 30 & 10 & 42 & 19 & 61 & $61 \%$ & 44 \\
\hline $\mathrm{DM}$ & 8 & 5 & 6 & 2 & 14 & 7 & 21 & $21 \%$ & 23 \\
\hline Hyperlipidemia & 10 & 4 & 4 & 2 & 14 & 6 & 20 & $20 \%$ & 28 \\
\hline Smoking & 18 & 0 & 5 & 0 & 23 & 0 & 23 & $23 \%$ & 52 \\
\hline Alcohol & 16 & 1 & 14 & 2 & 30 & 3 & 33 & $33 \%$ & - \\
\hline H/o Stroke/TIA & 5 & 1 & 3 & 0 & 9 & 1 & 9 & $9 \%$ & 10 \\
\hline H/o IHD/MI & 2 & 1 & 0 & 0 & 2 & 1 & 3 & $3 \%$ & 12 \\
\hline Atrial fibrillation & 4 & 2 & 0 & 0 & 4 & 2 & 6 & $6 \%$ & \\
\hline
\end{tabular}

Table - 2Age wise distribution of cerebrovascular stroke:

\begin{tabular}{|l|l|l|l|l|}
\hline \multirow{2}{*}{ Age } & \multicolumn{2}{|l|}{ Hemorrhages } & \multicolumn{2}{l|}{ Infarction } \\
\cline { 2 - 5 } & M & F & M & F \\
\hline $20-29$ & 0 & 0 & 0 & 0 \\
\hline $30-39$ & 1 & 0 & 0 & 0 \\
\hline $40-49$ & 9 & 3 & 5 & 3 \\
\hline $50-59$ & 14 & 9 & 8 & 4 \\
\hline $60-69$ & 11 & 3 & 7 & 6 \\
\hline $70-79$ & 5 & 1 & 4 & 5 \\
\hline $80-89$ & 0 & 0 & 1 & 1 \\
\hline $90-99$ & 0 & 0 & 0 & 0 \\
\hline
\end{tabular}

Table-3Incidence of pathological lesion of cerebrovascular disease in Tribal and non Tribal population

\begin{tabular}{|l|l|l|l|l|l|l|l|}
\hline \multirow{2}{*}{ Etiology } & \multicolumn{2}{l}{$\begin{array}{l}\text { Tribal } \\
\text { population }\end{array}$} & \multicolumn{2}{l|}{$\begin{array}{l}\text { Non tribal } \\
\text { population }\end{array}$} & \multicolumn{2}{l|}{ Total } & \multicolumn{2}{l|}{ Percentage } \\
\cline { 2 - 5 } \cline { 7 - 8 } & $\mathrm{M}$ & $\mathrm{F}$ & $\mathrm{M}$ & $\mathrm{F}$ & & Tribal & Non -tribal \\
\hline 1. Infarct & 11 & 7 & 14 & 12 & 44 & $36 \%$ & $52 \%$ \\
\hline 2.hemorrhage & 24 & 8 & 16 & 8 & 56 & $64 \%$ & $48 \%$ \\
\hline Total & 35 & 15 & 30 & 20 & 100 & $100 \%$ & $100 \%$ \\
\hline
\end{tabular}

Table-4 Survival among patients of cerebrovascular infarct in Tribal and non tribal population

\begin{tabular}{|l|l|l|l|l|l|l|}
\hline & M & F & \multicolumn{2}{|c|}{ Death } & \multicolumn{2}{c|}{ Survival } \\
\cline { 4 - 8 } & & & M & F & No & $\%$ \\
\hline Tribal & 11 & 7 & 1 & 3 & 14 & $78 \%$ \\
\hline Non Tribal & 14 & 12 & 2 & 2 & 22 & $85 \%$ \\
\hline Total & 25 & 19 & 3 & 5 & 36 & $82 \%$ \\
\hline
\end{tabular}

Table-5 Survival among patients of cerebrovascular hemorrhage in tribal and non tribal population

\begin{tabular}{|c|c|c|c|c|c|c|}
\hline & \multirow[t]{2}{*}{$\mathrm{M}$} & \multirow[t]{2}{*}{$\mathrm{F}$} & \multicolumn{2}{|c|}{ Death } & \multicolumn{2}{|c|}{ Survival \% } \\
\hline & & & $\mathrm{M}$ & $\mathrm{F}$ & No & $\%$ \\
\hline Tribal & 24 & 8 & 13 & 5 & 14 & $43 \%$ \\
\hline Non Tribal & 16 & 8 & 6 & 3 & 15 & $62 \%$ \\
\hline Total & 40 & 16 & 19 & 8 & 29 & $52 \%$ \\
\hline
\end{tabular}

\section{Discussion}


100 cases (50 Tribal and 50 Non Tribal) of cerebrovascular stroke were studied.Table 3 shows44 patients(44\%) had cerebral infarction,among these 18 were Tribal patient (11 male and 7 female) and 26 were non Tribal patient.In the infarct subgroup 12 patients were of age between 50-59 years $(\mathrm{M}=8, \mathrm{~F}=4)$ and 13 patients were between 60-69 years.(Table 2)56 patients (56\%) had a hemorrhagic stroke among which 32 were Tribal patients (24 male and 8 female) and 24 were non Tribal patients (Table 3 ). In the hemorrhagic subgroup 23 patients were of age 50-59years $(\mathrm{M}=18, \mathrm{~F}=7)$ and 14patients were age 60-69 years $(\mathrm{M}=24, \mathrm{~F}=10)$ Table 2.In the present study cerebrovascular strokes was more common in males(65\%) than females(35\%) which is compared to studies by aiyar et al who found $74 \%$ in male.Table 1Table 1 and 2 concludes that in the elderly age group (i.e. $>50$ years) the stroke is more prevalent (it consist of $79 \%$ of total patients) and more common in males $(65 \%)$ than females which is comparable to studies by aiyar et al who found $64 \%$ to be more than 50 years and L.pinhero et al who found $78 \%$ to be more than 50 years.

In this study group hemorrhagic strokes (56\%) are more common than ischemic stroke (44)which is different than Aiyer et al who found infarction in $70 \%$ cases, Sotaniemi k.A. et al found $66.2 \%$ infarct and $33.8 \%$ haemorrhages). Most common risk factor was hypertension $61 \%$, followed by alcoholism $33 \%$,smoking $23 \%$, hyperlipidemia20\% and diabetes $21 \%$. Table 1 and Table 3Most common etiology in Tribal patients was cerebral haemorrhages $(64 \%)$ while in non Tribal patients it was cerebral infarction(52\%).RELATION

\section{Between Cardiac Factor And Stroke:}

Out of 100 patient 4 patients had valvular heart disease, 2 patients had ischemic heart disease while out of 4 valvular patients 3 had atrial fibrillation. This conclude that cardiac factor plays an important role as a risk factor in cerebrovasculer disease it compare with the study of kaur et al 9 which showed $3 \%$ cases of rheumatic heart disease.

\section{Mortality in stroke:}

In the present study 35 (35\%) patients died. Mortality among Tribal patient due to ischemic and hemorrhagic stroke was $28 \%$ and $57 \%$ respectively and in non Tribal it was $15 \%$ and $48 \%$ respectively. Mortality among Tribal population was more compared to Non Tribals due to uncontrolled or undiagnosed hypertension, lack of education and awareness, use of alternative medication,poor compliance to therapy,lack of accessibility to health care facilities and their ignorance towards disease. Effective risk factor intervention and spreading awareness offers a real hope of reducing stroke mortality and morbidity in rural population.

\section{Conclusion}

Advancing age, male gender, hypertension, diabetes mellitus, smoking, dyslipidemia, alcohol consumption, heart disease, family history of stroke, and past history of Transient IschemicA or stroke were identified as risk factors for Cerebral stroke.Increasing age, male sex, low GCS at the time of admission, hypertension, diabetes mellitus, dyslipidemia, heart disease, smoking, presence of multiple risk factors, MCA territory infarct, presence of transtentorial herniation, intraparenchymal bleed with intraventricular extension were associated with poor prognosis.Hypertension is the most common risk factor followed by alcoholism.In this study hemorrhagicstroke was more common than Ischemic stroke and was associated with poor prognosis.In Tribal population hemorrhagic strokes were common while in Non Tribal,ischemic strokes were seen more commonly.Overall mortality was 35\%. Mortality among Tribal population was more compared to Non Tribals due to uncontrolled or undiagnosed hypertension, use of alternative medication,poor compliance to therapy,lack of accessibility to health care facilities and their ignorance.Public awareness of the risk factor(especially hypertension)and their management may lead to primary prevention of Cerebrovasculer accident. Early hospitalization of patients and their management reduces the mortality and disability in CVA.In India, particularly in rural areas, the health care delivery system is still deficient, both in quality and coverage. Accurate and current census data is not available, and the number of well trained physicians and health workers are limited, rendering the proper study of neuroepidemiology of cerebrovasculer disease difficult. As we do not know the real burden of various diseases, planning of health services and distribution of resources is difficult and at times an educated guess is the best.

\section{References}

[1]. Adams and Victor's principal of neurology $10^{\text {th }}$ edition chapter 34 Cerebrovasculer disease pg no 778-874.

[2]. Murray CJL and Lopez AD.The global burden of disease. 1. 1996. Harvard school of public health.

[3]. Aiyer et al: A study of clinicoradiological correlation in cerebrovasculer stroke (A study of 50 cases)Gujrat medical journal. vol. 52 march 1999.

[4]. Gauri et al: A study of risk factor and clinical profile of stroke at Bikaner.journal of API vol 4, 24jan 2000.

[5]. Pinhero L,damodar S et al .Risk factor in stroke.A prospective study: JAPI vol.48 jan 2000.

[6]. Sorganvi V, Kulkarni MS, Kadeli D, Atharga S; Risk Factors for Stroke: A Case Control Study. IJCRR, 2014 ; 6: 46-52. 
[7]. Singh RF, Suh IF, Singh VF, Chaithiraphan SF, Laothavorn PF, Sy RF et al. - Hypertension and stroke in Asia: prevalence, control and strategies in developing countries for prevention. - J Hum Hypertens 2000 Oct-Nov;14(10-11):749-63.749-63.

[8]. World Health Organization. The World Health Report: 2002: Reducing risks, promoting healthy life. 2002. World Health Organization.

[9]. Devichand and Caroli R K :A study of cerebrovascular strokes : J Indian med. Asso. Volm 36, no 121991

[10]. Miller M, Stone NJ, Ballantyne C, Bittner V, Criqui MH, Ginsberg HN et al.; Triglycerides and cardiovascular disease: a scientific statement from the American Heart Assosciation. Circulation, 2011; 123(20): 2292-2333.

[11]. Harrison's principles of internal medicine $19^{\text {th }}$ edition volume 2 chapter 446 Cerebrovasculer disease pg no2559-2586.

[12]. Gorelick PB; Alcohol and stroke. Stroke, 1987; 18: 268-271.

[13]. Broderick; Relationship of cardiac disease to stroke occurrence, recurrence and mortality. Stroke, 1992; 23: 1250-1256.

[14]. Howard G, Wagenknecht LE, Burke GL, DiezRoux A, Evans GW, Mc Govern P et al.; Cigarette smoking and progression of atherosclerosis: the Atherosclerosis Risk in Communities (ARIC) Study. JAMA, 1998; 279(2): 119-124. 